\title{
Hongos micorrícicos arbusculares (HMA) asociados a poblaciones naturales de Peumus boldus en Chile Central
}

\section{Arbuscular mycorrhizal fungi (AMF) linked to Peumus boldus natural formation in Central Chile}

\author{
Susana Benedetti ${ }^{1}$, Francisco Balocch $^{2 *} \&$ Marco Hormazábal $^{1}$ \\ ${ }^{1}$ Instituto Forestal, Av. Sucre 2397, Código Postal 7770223, Santiago, Chile. \\ Bioforest S.A., Camino a Coronel km 15, código postal 4190000, Coronel, Chile. \\ *francisco.balocchi@arauco.cl
}

\begin{abstract}
RESUMEN
Es conocido el aporte positivo a la vegetación que tiene la presencia de micorrizas arbusculares en el suelo a través de una relación simbiótica. La regeneración y establecimiento de Peumus boldus, boldo, especie endémica de mayor importancia económica y ambiental de Chile, es complicada, por lo que la identificación de hongos micorrícicos arbusculares (HMA) representa un importante aporte a la recuperación de formaciones naturales, así como al establecimiento de plantaciones de la especie. El presente estudio corresponde a una descripción cuantitativa de HMA presentes en formaciones naturales de Peumus boldus en Chile Central. Se identificaron 23 especies de HMA de las familias Acaulosporaceae (Acaulospora), Entrophosporaceae (Entrophospora), Glomeraceae (Funneliformis, Glomus, Rhizophagus y Sclerocystis) y Paraglomeraceae (Paraglomus aff. laccatum). Dentro de las especies encontradas, se destacan a Funneliformis badius y Funneliformis constrictus cuya presencia se detectó en todos los sitios. Asimismo, el índice de Simpson muestra en general una alta dominancia de especies de HMA en seis sitios estudiados. El índice de Shannon, por su parte, indica que existe una baja diversidad de especies en los sitios estudiados. Finalmente, este estudio servirá como base para optimizar las técnicas de establecimiento de boldo, al conocer que tipos de HMA pueden mejorar el éxito de la sobrevivencia y desarrollo de la especie bajo esquema de plantación, así como de enriquecimiento y restauración en formaciones naturales de la especie y del bosque esclerófilo.
\end{abstract}

Palabras clave: Formación natural de boldo, phylum Glomeromycota, simbiosis micorrícica.

\begin{abstract}
The positive contribution of tree-related mycorrhizae in the soil to plant ecosystems is well known, through symbiotic relationships. The regeneration and establishment of Peumus boldus (boldo), an endemic species of major economic and environmental relevance in Chile, is a complicated natural process, and the identification of tree-related mycorrhizal fungi (AMF) represents an important contribution to the recovery of native forest formations. The present study is a quantitative description of AMF, within natural boldo formations in central Chile. Twenty-three AMF species of several families --Acaulosporaceae (Acaulospora), Entrophosporaceae (Entrophospora), Glomeraceae (Funneliformis, Glomus, Rhizophagus, and Sclerocystis) and Paraglomeraceae (Paraglomus aff. Laccatum) -- were identified. Among these species, Funneliformis badius and Funneliformis constrictus were present at all sites. Likewise, Simpson index generally shows a high dominance of AMF species at all six studied sites. Shannon index, however, indicated that there is low species diversity. This study will serve as a basis to optimize boldo's establishment techniques, by knowing which types of AMF can improve the success of the survival and development of the species, either in the form of commercial plantation or natural sclerophyllous forest.
\end{abstract}

KEYwORDS: Boldo natural fomation, phylum Glomeromycota, mycorrhizal symbiosis.

\section{INTRODUCCIÓN}

Es conocido el aporte positivo de los microorganismos en el suelo en controlar la tasa de erosión, movimiento de nutrientes, y en general a la salud de la planta (Castillo et al. 2016; Castañeda et al. 2015; Jeffries et al. 2003). En este sentido, los hongos micorrícicos arbusculares (HMA) son un importante factor que puede ayudar al crecimiento 
de la vegetación (Urcoviche et al. 2014). Entre otras cosas, son los encargados del movimiento de nutrientes desde el suelo hacia la planta (Carrillo et al. 1992), por lo que facilitan el crecimiento y salud vegetal ( $\mathrm{Hu} \&$ Rufty 2007). En particular, las micorrizas arbusculares son las de mayor espectro y se pueden encontrar en la mayoría de las formaciones biológicas (Plenchette \& Duponnois 2005, Turnau \& Haselwandter 2002). En el caso particular de Chile, existen contados estudios con respecto a los HMA (Castillo et al. 2016). Por ejemplo, Medina et al. (2015) estudiaron la composición de HMA nativos en plantas pioneras en un sistema de agua dulce-salada en la Región de la Araucanía, en donde a pesar de la baja riqueza encontrada (5 especies), se encontró una nueva especie Corymbiglomus pacificum Oehl, J. Medina, P. Cornejo, Sánchez-Castro, G.A. Silva \& Palenz (Medina et al. 2014) asociada a Ammophyla arenaria (L.) Link. Carrillo et al. (1992) realizaron un estudio de la simbiosis micorrícica en comunidades boscosas en el sur de Chile. Sin embargo, y pese a incluir al boldo en el estudio, fue en forma de comunidad con otras especies, por lo que no es posible saber con exactitud que micorrizas específicamente se pueden encontrar en formaciones naturales puras de esta especie. Por otro lado, Urcoviche et al. (2014) estudiaron en plantas de boldo cultivadas en camas calientes el contenido de micorrizas en cuanto a densidad de esporas y colonización de raíces. El estudio encontró 15 especies en una primera medición y 8 en una segunda, además de una baja dominancia y diversidad.

Por otra parte, existe un conocido deterioro del bosque nativo en Chile debido principalmente a la sobreexplotación del recurso (Lara et al. 2009), sumado a la actividad agrícola y el subsiguiente cambio de uso del suelo (Castañeda et al. 2015). En boldo, la regeneración es complicada debido a cambios en la composición y estructura de este tipo vegetal, dado su sobreexplotación y degradación. Si a esto se suma que los suelos en Chile son predominantemente Andisoles y Ultisoles, que presentan altos contenidos de fósforo, pero con una baja absorción por parte de las plantas, hacen que la simbiosis de otro tipo de organismos ayude al crecimiento vegetal (Borie \& Rubio 1999). Otro problema que afecta la regeneración y presencia de la especie es el producido por los incendios forestales, los cuales queman la vegetación y la litera del suelo, combustionando la materia orgánica, deteriorando la estructura del suelo, aumentando la densidad aparente y disminuyendo los nutrientes del suelo (Fernández et al. 2004, Llovet et al. 2009, Certini 2005).

Peumus boldus Molina, boldo, es una especie endémica de Chile. Su importancia radica en su alto valor comercial relacionado con la exportación de sus hojas y corteza debido a su interesante contenido de componentes bioactivos; alcaloides, flavonoides y aceites esenciales (Kubinova et al. 2001, Speisky \& Cassels 1994, Verdeguer et al. 2011). En Chile se distribuye desde la Región de Coquimbo ( $\left.30^{\circ} 20^{\prime} \mathrm{S}\right)$ hasta la Región de Los Lagos (41²0’S) (Doll et al. 2005), pasando de climas semiáridos a lluviosos.

Con el fin de conocer el contenido de micorrizas en formaciones naturales, a saber, del boldo, el presente estudio cuantificó e identificó la presencia de Hongos Micorrícicos Arbusculares (HMA, phylum Glomeromycota) en muestras de suelo en nueve formaciones naturales en la Región de O'Higgins

\section{MÉTODOS}

\section{AnTeCEDEnTES DeL SITIO}

La elección del área para el estudio se basó en la región con la mayor superficie de la especie dentro de su área de distribución. En este sentido, de acuerdo al catastro del bosque nativo realizado por la Corporación Nacional Forestal (CONAF), la mayor superficie la posee la Región de O'Higgins, con 166 mil ha. Esta región se sitúa entre los paralelos $33^{\circ} 51^{\prime}$ y $35^{\circ} 01^{\prime} \mathrm{S}$ de latitud sur y $72^{\circ} 02^{\prime}$ y $70^{\circ}$ $02^{\prime} \mathrm{O}$ de longitud oeste. Las precipitaciones varían entre los 700 a $1.500 \mathrm{~mm}$ anuales las que aumentan de norte a sur. Posee un clima mediterráneo y la temperatura media durante los meses fríos es de $7{ }^{\circ} \mathrm{C}$, y en verano de $20^{\circ} \mathrm{C}$.

\section{Selección de Sitios E Individuos}

Para la selección de los nueve sitios (Fig.1), se definieron tres transectos a nivel regional de manera de cubrir las zonas de mayor concentración de la especie en condición de formación pura o especie dominante. Se realizó una primera campaña de terreno de preselección de individuos sobresalientes y una segunda campaña de validación de los individuos preseleccionados. La preselección se hizo basada en criterios o parámetros biométricos, los que fueron cuali-cuantitativos, a saber, altura total, proyección de copa (diámetros N-S y E-O), porcentaje de distribución de masa foliar en el individuo, densidad de hojas, tamaño de hojas, sanidad y altura de fuste o rebrotes libres de ramas. Esta preselección de individuos consideró una muestra de 25 ejemplares por sitio, de entre los cuales se eligió al mejor individuo en términos de características de crecimiento, biomasa y sanidad. Se consideraron estas características dado el supuesto de los conocidos beneficios de los HMA en el crecimiento de la planta. La elección de un solo individuo respondió a que se buscaba, adicionalmente, la selección de individuos candidatos a plus o superiores, con miras a iniciar un programa de resguardo de germoplasma y de mejora genética

\section{DETERMINACIÓN DE MICORRIZAS (HMA) POR SITIO}

Se tomaron muestras de suelo bajo el área de copa y cercano a la base de los individuos seleccionados. En cada sitio se tomaron tres muestras recolectadas al azar, para obtener un kilo de suelo por individuo. Para ello, se removió la litera en los primeros $15-20 \mathrm{~cm}$ superiores del suelo y a 
aproximadamente $20-30 \mathrm{~cm}$ de la base del individuo. Las muestras fueron enviadas al Laboratorio de Micología y Micorrizas de la Universidad de Concepción, Chile.

La extracción y separación de esporas se realizó siguiendo a Brundrett et al. (1996). Cada una de las nueve muestras de suelo fue tamizada para eliminar piedras y restos vegetales, y se tomó una submuestra de 100 g. Posteriormente, se realizó un tamizado húmedo mediante una serie de tamices de distintas aperturas $(500,250,106$ y $45 \mu \mathrm{m})$. El material retenido en cada tamiz fue recuperado y puesto en una solución de sacarosa al 70\% más agua. Cada muestra fue centrifugada a $2300 \mathrm{rpm}$ por cinco minutos a temperatura ambiente, en donde la interfaz que contiene las esporas fue recuperada y observada bajo lupa estereoscópica (Olympus SZ40) y microscopio óptico (Leitz Dialux). A continuación se realizó un conteo e identificación de esporas totales de cada muestra. Mediante una placa Doncaster y en suspensión con agua destilada, cada muestra fue observada bajo lupa estereoscópica para aislar todas las esporas y separarlas según diferencias en tamaño, color y textura. Para el conteo e identificación taxonómica, todas las esporas fueron montadas en portaobjetos con PVLG (alcohol polivinílicoácido láctico-glicerol) y reactivo de Melzer con el fin de observar las reacciones características de los distintos componentes de las paredes de la espora (Blaszkowski 2012, Brundrett et al. 1996). En relación a la determinación de características de las esporas, se siguió a Blaszkowski (2012); para la asignación de nombres se utilizó como referencia a Schüßler \& Walker (2010). Submuestras de 20 g fueron secadas en estufa a $60{ }^{\circ} \mathrm{C}$ durante una semana, con el objetivo de determinar el porcentaje de humedad y así expresar el número de esporas en $100 \mathrm{~g}$ de suelo seco.

ÍNDICES DE DOMINANCIA Y DIVERSIDAD DE HMA

Es común el uso de índices para comparar la diversidad de HMA (Moreno 2001). Para ello se utilizó el índice de dominancia de Simpson (Simpson 1949), en donde valores cercanos a cero indican la dominancia de alguna especie. Asimismo, se calculó el índice de riqueza de Shannon (Shannon \& Weaver 1949), el cual indica la variabilidad de especies, cuyos valores pueden alcanzar los 0,45 en suelos de cultivo hasta 3 en ecosistemas boscosos (Daniell et al. 2001, Becerra et al. 2011). Ambos índices se calcularon de acuerdo a las siguientes ecuaciones:

Índice de Shannon:

$$
H=-\sum_{s} p_{s} \cdot \log \left(p_{s}\right)
$$

Índice de Simpson:

$$
C=1-\sum_{s} p_{s}^{2}
$$

donde $\mathrm{p}_{\mathrm{s}}$ es la abundancia relativa de la especie $\mathrm{s}$ en la muestra sobre el total de especies en la muestra.
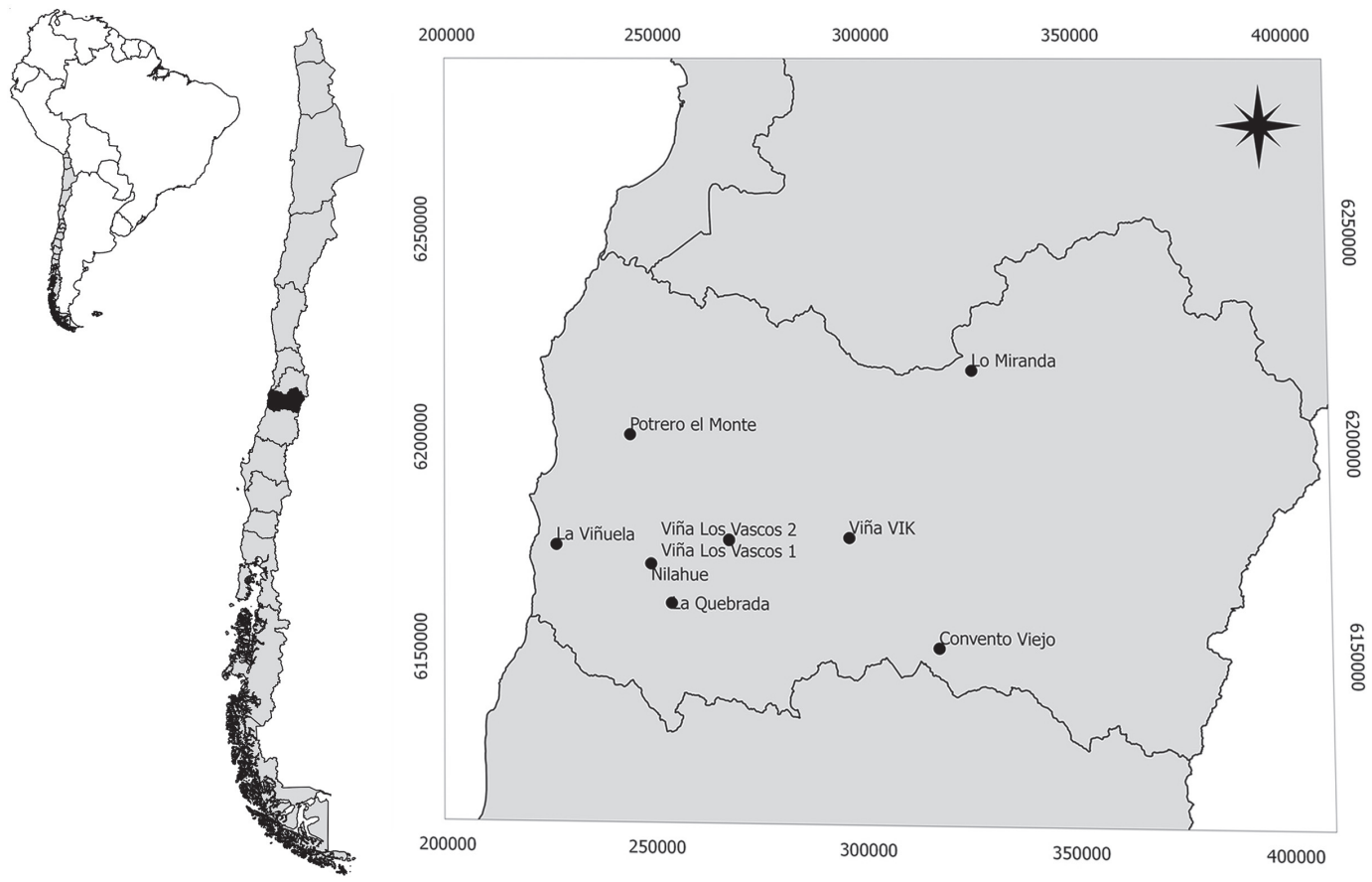

FIgURA 1. Región en estudio y puntos de muestreo de micorrizas, Región de O’Higgins. / Study site and sample AMF points, O’Higgins Region. 


\section{RESULTADOS}

Considerando el total de las muestras de suelo analizadas, se observaron 23 especies de HMA (Tabla 1) pertenecientes a las familias Acaulosporaceae (Acaulospora), Entrophosporaceae (Entrophospora), Glomeraceae (Funneliformis, Glomus, Rhizophagus y Sclerocystis) y Paraglomeraceae (Paraglomus aff. laccatum), cuya representación es variable en cada una de las muestras.

Dentro de cada sitio, el contenido de especies varió de 5 a 14 (Viña Los Vascos 1 y La Quebrada respectivamente, Tabla 1), con un promedio de 10 especies por sitio. Un dato importante de mencionar es que sólo tres sitios concentraron la mayor cantidad de especies, a saber: Potrero el Monte con un 56\% (13 especies), Convento Viejo con un 52\% (12 especies) y la Quebrada con un 62\% (14 especies). Además, estos 3 sitios comparten cinco especies, a saber: Acaulospora spinosa C. Walker \& Trappe, Funneliformis badius (Oehl, D. Redecker \& Sieverd.) C. Walker \& A. Schüssler, Funneliformis constrictus (Trappe) C. Walker \& A. Schüssler, Funneliformis mosseae (T.H. Nicolson \&
Gerd.) C. Walker \& A.Schüssler y Glomus microaggregatum (Koske, Gemma \& P.D. Olexia).

Cabe destacar la presencia de dos especies a lo largo de los nueve sitios (100\% presencia): F. badius (G.badium) y $F$. constrictus. Le siguen con una alta presencia $F$. mosseae con un $88 \%$, y A. spinosa y Rhizophagus irregularis (Błaszk., Wubet, Renker \& Buscot) C. Walker \& A. Schüssler, con un $77 \%$. Una particularidad sucede con F. badius cuyo conteo de esporas (abundancia) superó con creces al resto de las especies (2.768 esporas versus la menor con sólo 14). Además, es la especie con la mayor abundancia (número de esporas por sitio, 1.357 en Viña Los Vascos 1) en comparación a las otras especies reconocidas (e.g., Acaulospora lacunosa J.B.Morton en Viña Los Vascos 2 con sólo dos esporas).

En términos de dominancia, es posible observar diferencias entre los sitios estudiados (Tabla 2). En general, se observó una baja dominancia en seis sitios con valores entre 0,753 y 0,816 , en donde valores cercanos a uno indican una baja dominancia (o alta diversidad). El sitio Viña Los Vascos 1, por su parte, obtuvo el menor valor de

TABLA 1. Número de esporas por especies de HMA encontradas por sitio. / Number of spores per AMF species, found per site.

\begin{tabular}{|c|c|c|c|c|c|c|c|c|c|}
\hline ESPECIE & $\begin{array}{l}\text { VIÑa Los } \\
\text { VAsCos } 1\end{array}$ & $\begin{array}{l}\text { VIÑa Los } \\
\text { VAscos } 2\end{array}$ & Nitahue & $\begin{array}{l}\text { Potrero } \\
\text { eL MonTE }\end{array}$ & $\begin{array}{l}\text { VIÑA } \\
\text { VIK }\end{array}$ & $\begin{array}{c}\text { Convento } \\
\text { VIEJo }\end{array}$ & $\begin{array}{c}\text { LA } \\
\text { QUEBRADA }\end{array}$ & $\begin{array}{c}\text { LA } \\
\text { VIÑUELA }\end{array}$ & $\begin{array}{c}\text { Lo } \\
\text { MIRANDA }\end{array}$ \\
\hline Acaulospora lacunosa & & 2 & & 5 & & 7 & & & \\
\hline Acaulospora mellea & & & & 20 & 2 & 7 & & & 2 \\
\hline Acaulospora morrowiae & & & & & & & 14 & & \\
\hline Acaulospora scrobiculata & & & & & & & & 60 & \\
\hline Acaulospora spinosa & & 32 & 4 & 27 & 9 & 39 & 485 & 83 & \\
\hline Acaulospora tuberculata & & & & & & 2 & & 9 & 4 \\
\hline Entrophospora infrequens & 7 & & & & & 2 & 57 & & 2 \\
\hline Funneliformis badius & 1357 & 113 & 39 & 594 & 97 & 98 & 118 & 317 & 35 \\
\hline Funneliformis constrictus & 9 & 7 & 9 & 18 & 11 & 16 & 17 & 20 & 4 \\
\hline Funneliformis geosporus & & & & 113 & 22 & & 57 & 125 & 23 \\
\hline Funneliformis mosseae & 20 & & 11 & 25 & 34 & 114 & 107 & 49 & 10 \\
\hline Glomus aggregatum & & & 26 & & & & & 31 & \\
\hline Glomus albidum & & 2 & & 32 & & 32 & & & \\
\hline Glomus glomerulatum & & & & 84 & & & 33 & & \\
\hline Glomus macrocarpum & & 5 & & & 9 & & & & 19 \\
\hline Glomus microaggregatum & & 2 & & 27 & & 7 & 12 & 20 & \\
\hline Glomus multiforum & 47 & & 9 & & & 32 & 9 & & \\
\hline Glomus pustulatum & & & & 38 & & & 2 & & \\
\hline Rhizophagus diaphanus & & & & & 22 & & 31 & & \\
\hline Rhizophagus intraradices & & & 4 & 36 & & & 24 & & 6 \\
\hline Rhizophagus irregularis & & 18 & 68 & 5 & 27 & & 26 & 194 & 6 \\
\hline Sclerocystis sinuosa & & & & & 34 & 201 & & & \\
\hline Paraglomus aff. laccatum & & & & & & & & & 48 \\
\hline
\end{tabular}


Hongos micorrícicos arbusculares (HMA) en Peumus boldus: Benedetti, S. ET AL.

dominancia, cuya explicación es debido a que de las cinco especies encontradas en el sitio, F. badius concentra el $94 \%$ de esporas. Con respecto a la diversidad, ningún sitio obtuvo un valor mayor a dos, situación esperable debido a la alta dominancia de algunas especies. En relación al sitio, Viña Los Vascos 1 obtuvo el menor valor de diversidad, situación explicada por la dominancia de la especie $F$. badius mencionada anteriormente.

TABLA 2. Índices de Shannon y Simpson por sitio. / Shannon and Simpson indexes per study site.

\begin{tabular}{|c|c|c|c|c|c|c|c|c|c|}
\hline Sitio & $\begin{array}{l}\text { VIÑa Los } \\
\text { VASCOS } 1\end{array}$ & $\begin{array}{l}\text { VIÑA LoS } \\
\text { VASCOS } 2\end{array}$ & NiLAhUE & $\begin{array}{c}\text { Potrero el } \\
\text { Monte }\end{array}$ & VIÑA VIK & $\begin{array}{c}\text { ConVEnTo } \\
\text { VIEJO }\end{array}$ & $\begin{array}{c}\text { LA } \\
\text { QuEBRADA }\end{array}$ & $\begin{array}{c}\text { LA } \\
\text { VIÑUELA }\end{array}$ & $\begin{array}{c}\text { Lo } \\
\text { MIRANDA }\end{array}$ \\
\hline Simpson & 0,110635 & 0,566466 & 0,75308 & 0,638289 & 0,807754 & 0,784106 & 0,724498 & 0,795647 & 0,816819 \\
\hline Shannon & 0,284654 & 1,204284 & 1,656191 & 1,594742 & 1,932394 & 1,820048 & 1,816994 & 1,855014 & 1,944971 \\
\hline
\end{tabular}

\section{DISCUSIÓN}

El contenido de HMA en el bosque nativo en Chile es conocido (Moreno-Chacón \& Lusk 2004, Borie et al. 2010, Castillo et al. 2012, Torres-Mellado et al. 2012, Castillo et al.2016), por lo que saber que especies están asociadas a los bosques degradados es de suma importancia, considerando que esta condición de degradación está siendo cada vez más frecuente en el país. Esto es relevante, especialmente cuando no existen muchos estudios ligados al contenido de estos hongos en el bosque esclerófilo, formación vegetacional con alto grado de degradación. En general, en el presente estudio se visualizan géneros similares de HMA de acuerdo a otros estudios de HMA en boldo (e.g., Urcoviche et al. 2014).

Debido a la conocida amplitud del género Glomus en variados tipos de hábitats (Sýkorová et al. 2007), su presencia es esperable, aún más cuando es el primer género en la sucesión en un sitio (Oehl et al. 2004), además de ser uno de los géneros con mayor resistencia a cambios en el ecosistema como por ejemplo disturbios en el suelo (Zangaro $\&$ Moreira 2010). Cabe destacar la variabilidad existente en el contenido de HMA en las distintas situaciones analizadas. Un ejemplo son los sitios Viñas Los Vascos 1 y 2, los que se encuentran distanciados a solo $40 \mathrm{~m}$ entre ellos. Es decir, las mismas condiciones edafoclimáticas, pero una composición diferente en cuanto a especies de HMA.

Entre los siete géneros de HMA encontrados en las muestras, Funneliformis fue el con mayor presencia (Tabla 3, 7 de 9 sitios). No se visualiza una codominancia importante de otra especie en los sitios estudiados, donde la distribución de especies fue heterogénea. Es importante señalar a la familia Glomeraceae con una presencia promedio del 56\% en todos los sitios muestreados (e.g., máximo de $96 \%$ en Villa Los Vascos 1). En este sentido, Dodd et al. (2000) encontraron que el contenido de HMA en una raíz puede ser variado. Esta situación pudo ser corroborada con los resultados mostrados anteriormente en donde es posible encontrar 23 especies diferentes en raíces de boldo. Pereira et al. (2014) encontraron 30 especies de HMA en un bosque tropical del atlántico y Husband et al. (2002), 29 especies en tres sitios en Panamá. Esta situación permite inferir que la concentración de especies en boldo es comparable con aquella de bosques altamente complejos y de diferente régimen hídrico. Sin embargo, una gran diferencia con biotas tropicales es la presencia del género Acaulospora (Trufem \& Viriato 1990, Guadarrama \& Alvarez-Sánchez 1999), la que es común de encontrar en esos sitios, no así en el presente estudio con un clima templado a semiárido.

Dado los antecedentes presentados y en general, boldo prefiere especies de los géneros Glomeraceae, específicamente del género Funneliformis. En particular con F. mosseae y F. geosporus, su alta presencia se sustenta por ser una de las especies de mayor importancia ecológica y económica, la cual es posible de encontrar tanto en ambientes no alterados como en áreas con disturbios antrópicos y en diferentes climas y hábitats (Nadimi et al. 2016). Además y conjuntamente con F. badius (G. badium), es común encontrarlos en suelos neutros a alcalinos $(\mathrm{pH}$ 6-8) (Oehl et al. 2005). Por ejemplo, Zangaro et al. (2013) estudiaron la evolución de HMA en un bosque lluvioso en Brasil, encontrando a $F$. mosseae, $F$. geosporus y $F$. constrictus en los 4 sitios estudiados (pastizal, matorral, bosque secundario y bosque maduro semicaducifolio) con una frecuencia de ocurrencia del 100\%, situación que complementa la amplitud de estas especies.

Esta situación permitiría inferir que el boldo es altamente dependiente de este tipo de hongos, situación que Urcoviche et al. (2014) menciona en su estudio de contenido de HMA en algunas plantas vasculares medicinales y en el cual incluyeron al boldo. De hecho, del total de especies encontradas en el estudio anteriormente mencionado, solo dos de ellas coinciden con el presente estudio. Es decir, no sólo es esperable que el contenido de HMA en el suelo sea beneficioso para el boldo, si no 
que parece ser un importante factor que puede aportar al establecimiento de otras formaciones vegetacionales. Por ejemplo, es sabido el rol de los HMA en el mejoramiento de los agregados en el suelo (e.g., estudio en un suelo vertisol por Bearden \& Petersen 2000).

Es interesante rescatar que el número de especies encontradas, en general, es bastante alto y es comparable con cantidades encontradas en sitios con horticultura y en cultivos de trigo, y en especial de la especie Funneliformis spp., la cual es poco común (Castillo et al. 2016). Sin embargo, sería relevante comprobar que la asociación de algunos géneros y los cuales se vieron diferenciados en los nueve sitios, sean estudiados de manera más intensiva con el fin de lograr un estudio acabado de la regeneración y/o restauración de boldo en Chile, por un lado, y por otro investigar la simbiosis de boldo con otras especies vasculares y así poder contrarrestar de alguna manera el deterioro del bosque nativo chileno. Finalmente, el contenido de hongos micorrícicos arbusculares muestra las pautas de una futura recuperación y regeneración de boldo en Chile, presentando en general una importante diversidad de especies de HMA y una baja dominancia en comparación a otros estudios (e.g., Urcoviche et al. 2014).

TABLA 3. Frecuencia relativa reperesentada en porcentaje (\%) por géneros de HMA. / Relative frecuency represented in percentage (\%) by genus of HMA.

\begin{tabular}{|c|c|c|c|c|c|c|c|c|c|}
\hline \multirow[b]{2}{*}{ GÉNERO } & \multicolumn{9}{|c|}{ FRECUENCIA RELATIVA (\%) } \\
\hline & $\begin{array}{l}\text { VIÑA Los } \\
\text { VASCOS } 1 \\
\end{array}$ & $\begin{array}{l}\text { VIÑA LoS } \\
\text { VASCOS } 2 \\
\end{array}$ & Nilahue & $\begin{array}{c}\text { Potrero } \\
\text { EL Monte }\end{array}$ & $\begin{array}{l}\text { VIÑA } \\
\text { VIK }\end{array}$ & $\begin{array}{c}\text { Convento } \\
\text { VIEJo } \\
\end{array}$ & $\begin{array}{c}\text { LA } \\
\text { QuEBRADA }\end{array}$ & $\begin{array}{c}\text { LA } \\
\text { VIÑUELA }\end{array}$ & $\begin{array}{c}\text { Lo } \\
\text { MIRANDA }\end{array}$ \\
\hline Acaulospora & 0,00 & 18,78 & 2,35 & 5,08 & 4,12 & 9,87 & 50,30 & 16,74 & 3,77 \\
\hline Entrophospora & 0,49 & 0,00 & 0,00 & 0,00 & 0,00 & 0,36 & 5,75 & 0,00 & 1,26 \\
\hline Funneliformis & 96,25 & 66,30 & 34,71 & 73,24 & 61,42 & 40,93 & 30,14 & 56,28 & 45,28 \\
\hline Glomus & 3,26 & 4,97 & 20,59 & 17,68 & 3,37 & 12,75 & 5,65 & 5,62 & 11,95 \\
\hline Rhizophagus & 0,00 & 9,94 & 42,35 & 4,00 & 18,35 & 0,00 & 8,17 & 21,37 & 7,55 \\
\hline Sclerocystis & 0,00 & 0,00 & 0,00 & 0,00 & 12,73 & 36,09 & 0,00 & 0,00 & 0,00 \\
\hline Paraglomus & 0,00 & 0,00 & 0,00 & 0,00 & 0,00 & 0,00 & 0,00 & 0,00 & 30,19 \\
\hline
\end{tabular}

\section{REFERENCIAS}

Bearden, B.N., Petersen, L. 2000. Influence of arbuscular mycorrhizal fungi on soil structure and aggregate stability of a vertisol. Plant and Soil 218(1-2): 173-183.

Becerra, A.G., Cabello, M.N., Bartoloni, N.J. 2011. Native arbuscular mycorrhizal fungi in the Yungas forests, Argentina. Mycologia 103: 273-279.

Blaszkowski, J. 2012. Glomeromycota. W. Szafer Institute of Botany. Polish Academy of Sciences, Cracow, Poland. 303 pp.

Borie, F., Rubio, R. 1999. Effects of arbuscular mycorrhizae and liming on growth and mineral acquisition of aluminumtolerant and aluminum-sensitive barley cultivars. Journal of Plant Nutrition 22(1): 121-137.

Borie, F., Rubio, R., Morales, A., Curaqueo, G., Cornejo, P. 2010. Arbuscular mycorrhizae in agricultural and forest ecosystems in Chile. Journal of Soil Science and Plant Nutrition 10(3): 185-206.

Brundrett, M., Bougher, N., Dell, B., Grove, T., Malajczuk, N. 1996. Working with mycorrhizas in forestry and agriculture. Australian Centre for International Agricultural Research, Canberra, Australia. 344 pp.

Carrillo, R., Godoy, R., Peredo, H. 1992. Simbiosis micorrícica en comunidades boscosas del Valle Central en el sur de Chile. Bosque 13(2): 57-67.
Castañeda, L.E., Godoy, K., Manzano, M., Marquet, P.A., BARbosa, O. 2015. Comparison of soil microbial communities inhabiting vineyards and native sclerophyllous forests in central Chile. Ecology and evolution 5(18): 3857-3868.

Castillo, C.G., Borie, F., Godoy, R., Rubio, R., Sieverding, E. 2012. Diversity of mycorrhizal plant species and arbuscular mycorrhizal fungi in evergreen forest, deciduous forest and grassland ecosystems of Southern Chile. Journal of Applied Botany and Food Quality 80(1): 40-47.

Castillo, C.G., Borie, F., Oehl, F., Sieverding, E. 2016. Arbuscular mycorrhizal fungi biodiversity: prospecting in SouthernCentral zone of Chile. A review. Journal of Soil Science and Plant Nutrition 16(2): 400-422.

Certini, G. 2005. Effects of fire on properties of forest soils: a review. Oecologia 143(1): 1-10.

Daniell, T.J., Husband, R., Fitter, A.H., Young, J.P.W. 2001. Molecular diversity of arbuscular mycorrhizal fungi colonizing arable crops. FEMS Microbiology Ecology 36: 203-209.

Dodd, J.C., Boddington, C.L., Rodriguez, A., Gonzalez-Chavez, C., Mansur, I. 2000. Mycelium of arbuscular mycorrhizal fungi (AMF) from different genera: form, function and detection. Plant and Soil 226(2): 131-151.

Doll, U., Aedo, D., Lopez, P. 2005. Caracterización morfológica de tres procedencias de boldo (Peumus boldus) en una plantación joven de 6 años. Bosque 26(3): 45-54. 
Fernández, C., Vega, J.A., Gras, J.M., Fonturbel, T., Cuinas, P., Dambrine, E., Alonso, M. 2004. Soil erosion after Eucalyptus globulus clearcutting: differences between logging slash disposal treatments. Forest Ecology and Management 195(1): 85-95.

Guadarrama, P., Álvarez-Sánchez, F.J. 1999. Abundance of arbuscular mycorrhizal fungi spores in different environments in a tropical rain forest, Veracruz, Mexico. Mycorrhiza 8(5): 267-270.

Hu, S., RuFTy, T. 2007. Linking arbuscular mycorrhizal fungi with plant health: Mechanisms and challenges. Phytopathology 97(7):142.

Husband, R., Herre, E.A., Turner, S.L., Gallery, R., Young, J.P.W. 2002. Molecular diversity of arbuscular mycorrhizal fungi and patterns of host association over time and space in a tropical forest. Molecular Ecology 11(12): 2669-2678.

Jeffries, P., Gianinazzi, S., Perotto, S., Turnau, K., Barea, J.M. 2003. The contribution of arbuscular mycorrhizal fungi in sustainable maintenance of plant health and soil fertility. Biology and Fertility of Soils 37(1): 1-16.

Kubinova, R., Machala, M., Minksova, K., Neca, J., SuchÝ, V. 2001. Chemoprotective activity of boldine: modulation of drugmetabolizing enzymes. Die Pharmazie 56(3): 242-243.

Lara, A., Little, C., Urrutia, R., McPhee, J., Álvarez-Garretón, C., Oyarzún, C., Soto, D., Donoso, P., Nahuelhual, L., Pino, M., Arismendi, I. 2009. Assessment of ecosystem services as an opportunity for the conservation and management of native forests in Chile. Forest Ecology and Management 258(4): 415-424.

Llovet, J., Ruiz-Valera, M., Josa, R., Vallejo, V.R. 2009. Soil responses to fire in Mediterranean forest landscapes in relation to the previous stage of land abandonment. International Journal of Wildland Fire 18(2): 222-232.

Medina, J., Cornejo, P., Borie, F., Meier, S., Palenzuela, J., Vieira, H.E.E., Ferreira, A., Silva, G., Sánchez-Castro, I., Oehl, F. 2014. Corymbiglomus pacificum, a new glomeromycete from a saline lakeshore in Chile. Mycotaxon 127(1): 173183.

Medina, J., Meier, S., Rubio, R., Curaqueo, G., Borie, F., Aguilera, P., Oehl, F.,Cornejo, P. 2015. Arbuscular mycorrhizal status of pioneer plants from the mouth of lake Budi, Araucanía Region, Chile. Journal of Soil Science and Plant Nutrition 15(1): 142-152.

Moreno, C. 2001. Métodos para medir la biodiversidad. M6TManuales y Tesis. SEA, Zaragoza, 84 pp.

Moreno-Chacón, M., Lusk, C. H. 2004. Vertical distribution of fine root biomass of emergent Nothofagus dombeyi and its canopy associates in a Chilean temperate rainforest. Forest Ecology and Management 199(2): 177-181.

Nadimi, M., Stefani, F.O., HijRi, M. 2016. The large (134.9 $\mathrm{kb})$ mitochondrial genome of the glomeromycete Funneliformis mosseae. Mycorrhiza 26(7): 747-755.

Oehl, F., Redecker, D., Sieverding, E. 2005. Glomus badium, a new sporocarpic mycorrhizal fungal species from European grasslands with higher soil $\mathrm{pH}$. Journal of Applied Botany and Food Quality 79(1): 38-43.

Oehl, F., Sieverding, E., Mäder, P., Dubois, D., Ineichen, K., Boller, T., Wiemken, A. 2004. Impact of long-term conventional and organic farming on the diversity of arbuscular mycorrhizal fungi. Oecologia 138(4): 574-583.

Pereira, C.M.R., Da Silva, D.K.A., De Almeida Ferreira, A.C., Goto, B.T., MaiA, L.C. 2014. Diversity of arbuscular mycorrhizal fungi in Atlantic forest areas under different land uses. Agriculture, Ecosystems \& Environment 185: 245-252.

Plenchette, C., Duponnois, R. 2005. Growth response of the saltbush Atriplex nummularia L. to inoculation with the arbuscular mycorrhizal fungus Glomus intraradices. Journal of Arid Environments 61(4): 535-540.

SCHÜSSLER, A., WALKER, C. 2010. The Glomeromycota: a species list with new families and new genera. CreateSpace Independent Publishing Platform, Gloucester, England. 58 pp.

Shannon, C.E., Weaver, W. 1949. The mathematical theory of communication. University of Illinois Press, USA. $17 \mathrm{pp}$.

Simpson, E.H. 1949. Measurement of diversity. Nature 163: 688.

SPeisky , H., Cassels, B.K. 1994. Boldo and boldine: an emerging case of natural drug development. Pharmacological Research 29(1): 1-12.

Sýkorová, Z., Ineichen, K., Wiemken, A., Redecker, D. 2007. The cultivation bias: different communities of arbuscular mycorrhizal fungi detected in roots from the field, from bait plants transplanted to the field, and from a greenhouse trap experiment. Mycorrhiza 18(1): 1-14.

Torres-Mellado, G.A., Escobar, I., Palfner, G., CasanovaKATNY, M.A. 2012. Mycotrophy in Gilliesieae, a threatened and poorly known tribe of Alliaceae from central Chile. Revista Chilena de Historia Natural 85: 179-186.

Trufem, S.F.B., Viriato, A. 1990. Fungos micorrízicos vesículoarbusculares da Reserva Biológica do Alto da Serra de Paranapiacaba, São Paulo, Brasil. Revista Brasileira de Botânica 13: 49-54.

Turnau, K., Haselwandter, K. 2002. Arbuscular mycorrhizal fungi, an essential component of soil microflora in ecosystem restoration. In: Mycorrhizal Technology in Agriculture: From Genes to Bioproducts, pp. 137-149. Birkhäuser Basel, Switzerland.

Urcoviche, R.C., Castelli, M., Gimenes, R.E., Alberton, O. 2014. Spore density and diversity of arbuscular mycorrhizal fungi in medicinal and seasoning plants. African Journal of Agricultural Research 9(16): 1244-1251.

Verdeguer, M., García-Rellán, D., Boira, H., Pérez, E., Gandolfo, S., Blázquez, M.A. 2011. Herbicidal activity of Peumus boldus and Drimys winteri essential oils from Chile. Molecules 16(1): 403-411.

Zangaro, W., Moreira, M. 2010. Micorrizas arbusculares nos biomas floresta atlântica e floresta de araucária. En: Siqueira, J.O., Souza, F.A., Cardoso, E.J.B.N., Tsai, S.M. (eds.), Micorrizas: trinta anos de pesquisa no Brasil, pp. 279-310. Editora UFLA, Brasília, Brasil.

Zangaro, W., Rostirola, L.V., De Souza, P.B., De Almeida Alves, R., Lescano, L.E.A.M., Rondina, A.B.L., Nogueira, M.M., Carrenho, R. 2013. Root colonization and spore abundance of arbuscular mycorrhizal fungi in distinct successional stages from an Atlantic rainforest biome in southern Brazil. Mycorrhiza 23(3): 221-233.

Recibido: 10.10 .2017

Aceptado: 02.01.2018 